Cite this: Phys. Chem. Chem. Phys., 2013 15, 11402

Received 11th March 2013, Accepted 7th May 2013

DOI: $10.1039 /$ c3cp51057c

www.rsc.org/pccp

\section{Time-resolved photoelectron spectroscopy of adenine and adenosine in aqueous solution}

\author{
Franziska Buchner, Hans-Hermann Ritze, Jan Lahl† and Andrea Lübcke* $\ddagger$
}

\section{Introduction}

Interaction of ultraviolet light with DNA molecules may lead to photodamage and consequently may cause development of cancer. However, natural DNA bases are much less photoreactive than similar molecules. ${ }^{1}$ One reason for the high photostability of the DNA bases is their ultrafast electronic deactivation processes that prevent the molecules from severe changes such as bond breakage. The mechanisms of these ultrafast processes have been studied over the recent decade ${ }^{1-8}$ but are still not fully understood.

Motivated by recent developments in fs-liquid jet photoelectron spectroscopy, ${ }^{9,10}$ we used this technique to study the ultrafast radiationless relaxation mechanism of the DNA base adenine and its ribonucleoside adenosine in aqueous solution. The fs-photoelectron spectroscopy provides direct access to the (time-dependent) binding energy of the excited state and maps the excited state potential energy hyper surface onto the ionization continuum. Furthermore, the time-dependent photoelectron signal yields conclusions on the excited state population dynamics.

As the other DNA bases, adenine may exist in different tautomeric forms. As evident from theory ${ }^{11-13}$ and experiment, ${ }^{14-17}$ adenine shows up in aqueous solution in two coexisting tautomers: the $\mathbf{7} \boldsymbol{H}$-Ade and the $\mathbf{9 H}$-Ade. Fig. 1 shows the structure of these two tautomers. The excited state lifetimes for $\mathbf{9 H}$ - and $\mathbf{7 H}$-Ade in water are on the order of $0.2-0.6$ ps and $9 \mathrm{ps}$, respectively. ${ }^{18-20}$ In the adenosine molecule, the ribose moiety replaces the hydrogen at the N9-atom and therefore

Max-Born-Institut für nichtlineare Optik und Kurzzeitspektroskopie, Max-Born-Straße 2A, 12489 Berlin, Germany. E-mail: luebcke@mbi-berlin.de $\dagger$ Present address: TU Berlin, Institut für Optik und Atomare Physik, Hardenbergstraße 36, 10623 Berlin, Germany.

‡ Present address: Laboratoire de Spectroscopie Ultrarapide, EPFL, 1015 Lausanne, Switzerland.

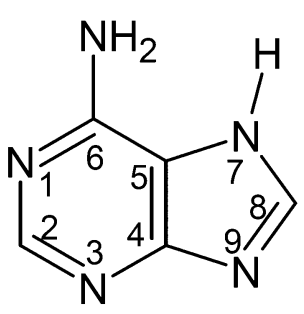

7H Ade<smiles>[R]n1cnc2c(N)ncnc21</smiles>

$9 H$ Ade $(\mathrm{R}=\mathrm{H})$

Ado ( $R=$ ribose)
Fig. 1 Structures of the $7 \mathrm{H}$ and $9 \mathrm{H}$ tautomer of adenine (Ade). In adenosine (Ado), the ribose replaces the hydrogen at position 9 and therefore prevents tautomerization.

prevents the formation of the $7 H$ tautomer. Correspondingly, the value for the excited state lifetime of adenosine was determined to be $0.3-0.5$ ps. ${ }^{18,20-22}$

Theoretical studies of isolated adenine (especially the $9 H$ tautomer) in the gas phase identified several conical intersections (CIs) relevant for the excited state relaxation $\left(\pi \pi^{*} / n \pi^{*}\right.$, $\left.\pi \pi^{*} / \pi \sigma^{*}, \mathrm{n} \pi^{*} / \mathrm{S}_{0}, \pi \pi^{*} / \mathrm{S}_{0}, \pi \sigma^{*} / \mathrm{S}_{0}\right)^{3-8,23-26}$ (and references therein). These CIs are connected with geometrical changes of the molecule including an envelope puckering at the $\mathrm{C} 2$-atom $\left({ }^{2} \mathrm{E}, \pi \pi^{*} / \mathrm{S}_{0}\right)$, a pronounced out-of-plane distortion of the C6-atom and the amino group $\left({ }^{1} \mathrm{~S}_{6}, \mathrm{n} \pi^{*} / \mathrm{S}_{0}\right)$, stretch of the N9-H bond $\left(\pi \sigma^{*} / \mathrm{S}_{0}\right)$ and/or C8-N9 bond breaking. The type of conical intersections was classified according to the scheme outlined in Fig. 1 of ref. 27. For excitation energies of around $5.0 \mathrm{eV}$ (the maximum of the absorption band in the gas phase), two mechanisms were found to explain the lifetimes observed in experiments: $\pi \pi^{*} \rightarrow \mathrm{S}_{0}$ via the ${ }^{2} \mathrm{E} \mathrm{CI}$ and $\pi \pi^{*} \rightarrow \mathrm{n} \pi^{*}$ are responsible for the ultrafast decay below $200 \mathrm{fs}$, whereas $\mathrm{n} \pi^{*} \rightarrow \mathrm{S}_{0}$ via the ${ }^{1} \mathrm{~S}_{6}$ CI 
explains the second, slightly slower lifetime below 1.0 ps. For excitation energies of around $4.7 \mathrm{eV}$ and above $5.3 \mathrm{eV}$, another channel arises: $\pi \pi^{*} \rightarrow \pi \sigma^{*} \rightarrow \mathrm{S}_{0}$ which is associated with hydrogen abstraction.

Theoretical studies of adenine in solution are rare because not only all the relevant electronic states have to be described realistically but also the effect of the surrounding molecules has to be included. There have been different approaches to include the effect of the environment on the relaxation of adenine. ${ }^{26,28-31}$ Mitric et al. used the time-dependent density functional tight binding method combined with a surface hopping algorithm to calculate non-adiabatic relaxation of microsolvated adenine. ${ }^{31}$ After excitation with $260 \mathrm{~nm}$ light into $S_{3}$, they found a 16 fs transition into $S_{1}$ and a time constant of $200 \mathrm{fs}$ for repopulation of the ground state. Lan et al. recently studied the non-adiabatic dynamics of $\mathbf{9 H}$-Ade in aqueous solution using surface-hopping quantum mechanical-molecular mechanical (QM/MM) simulations. ${ }^{26}$ They found a relaxation mechanism that also appears for the gas phase (a two-step $\pi \pi^{*} \rightarrow \mathrm{n} \pi^{*} \rightarrow \mathrm{S}_{0}$ pathway via the $\left.{ }^{1} \mathrm{~S}_{6}-\mathrm{CI}\right)$. Due to a slight nonplanarity of the molecule in solution (out-of-plane amino group), the molecule is preformed for the ${ }^{1} \mathrm{~S}_{6}$ conical intersection. Thus, this pathway is dynamically favored and dominates the relaxation. This also explains the faster relaxation in solution with respect to the gas phase.

Lan et al. also calculated the vertical excitation energies of the first three excited states and found $S_{1}$ between 4.45 and $4.69 \mathrm{eV}$, whereas $\mathrm{S}_{2}$ and $\mathrm{S}_{3}$ lie within $0.1 \mathrm{eV}$ at 4.69-4.95 eV. After excitation of $\mathrm{S}_{2}$, Lan et al. found an ultrafast population transfer into $S_{1}$ with a time constant of $40 \mathrm{fs}$ and a time constant of $410 \mathrm{fs}$ for internal conversion from $\mathrm{S}_{1}$ into the ground state.

For the second tautomer ( $7 \boldsymbol{H}$-Ade), there has been significantly less theoretical activity. Marian et al. found some higher potential barriers for the relaxation into the ground state for bare $\mathbf{7} \boldsymbol{H}$-Ade compared to $\mathbf{9 H}$-Ade. ${ }^{32}$ These barriers would explain the larger excited state lifetime assigned to $\mathbf{7 H}$-Ade in solution.

In Fig. 2, a schematic of the potential energy curves for the $9 H$ tautomer of adenine in water is presented. To define the relative position of the curves, experimental and theoretical values (orange) are used:

(1) UV/Vis spectra show that the absorption of adenine in water starts at about $4.3 \mathrm{eV}$. This is the lowest absorbable photon energy and therefore represents the 0-0 transition between the ground state $S_{0}$ and the first excited state $S_{1}$.

(2) The vertical ionization energy of adenine in aqueous solution was deduced experimentally to be $7.5 \mathrm{eV} .^{33}$

(3) The adiabatic ionization potential of adenosine in water has been calculated using ab initio methods together with the equilibrium polarizable continuum model to be about $6.6 \mathrm{eV} .^{34}$

The general shape of the excited state $S_{1}$ is adapted from Blancafort et al., who calculated the excited-state potential energy surface for different relaxation paths: ${ }^{35}$ in the region of vertical excitation from the ground state, the excited state $S_{1}$ is quite flat and bends downwards near the conical intersection (there are several conical intersections with the ground state; we did not pick any specific one). According to Lan et al., the

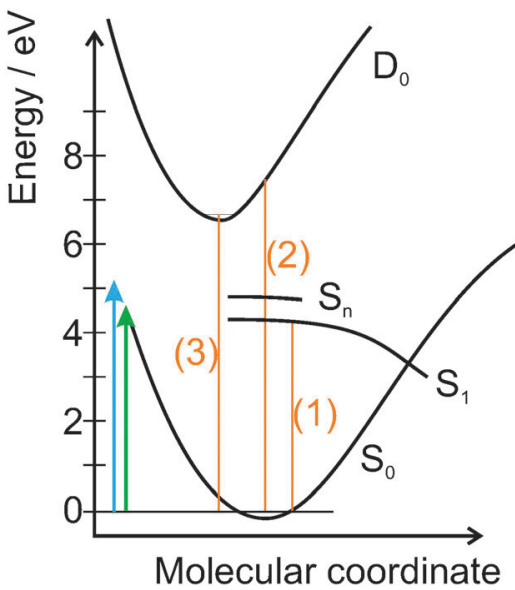

Fig. 2 Schematic representation of the potential energy curves of $9 \mathrm{H}$ tautomer of adenine in water, where $S_{0}$ is the ground state, $S_{1}$ the lowest limit of the excited state and $D_{0}$ the first ionic state. Higher excited states are referred to as $S_{n}$. The arrows indicate the photon energies of pump and probe pulses ( $4.66 \mathrm{eV}$ and $5.21 \mathrm{eV}$ ). The relative positions of the curves are determined by (1) the onset of the absorption spectrum, (2) the vertical ionization energy, ${ }^{33}$ and (3) the adiabatic ionization potential. ${ }^{34}$ For details see the text.

higher excited states $S_{2}$ and $S_{3}$ lie very close together, directly above $4.7 \mathrm{eV}^{26}$ We indicated these by $\mathrm{S}_{\mathrm{n}}$.

\section{Experiment}

We perform time-resolved photoelectron spectroscopy of liquid samples using the liquid jet technology. ${ }^{36}$ A liquid jet with a diameter of about $10 \mu \mathrm{m}$ is generated by pushing the sample solution through a thin fused silica nozzle into a vacuum using an HPLC pump. The jet velocity is about $50 \mathrm{~m} \mathrm{~s}^{-1}$. In the first few millimeters the jet flows laminarly before it breaks apart into droplets. The experiment is carried out at the laminar part of the jet.

A Ti:sapphire laser system delivers $40 \mathrm{fs}$ pulses at $800 \mathrm{~nm}$. One part of the laser output is used to pump a parametric optical generator (light conversion, TOPAS) and to deliver pulses between $238 \mathrm{~nm}(5.21 \mathrm{eV})$ and $248 \mathrm{~nm}(5.00 \mathrm{eV})$. The remaining laser output is used to generate light at $266 \mathrm{~nm}$ (4.66 eV) by sum frequency generation in BBO crystals. The UV pulses have a duration of $\sim 80 \mathrm{fs}$ and are attenuated to $40-50 \mathrm{~nJ}$ for the pump-probe experiment. With our pulse duration of about $80 \mathrm{fs}$, we will not resolve the fast transition from $S_{n}$ to $S_{1}$. Therefore, the observed dynamics should be dominated by the relaxation of $S_{1}$ into the ground state.

Adenine and adenosine significantly absorb light at all wavelengths used in this experiment. ${ }^{37}$ We therefore expect to observe excited state dynamics initiated by either UV pulse.

The pulses are focused onto the liquid jet to focal spot sizes of about $100 \mu \mathrm{m}$. A chopper in each beam path allows us to separately measure and subtract the one-color multi-photon signals (pump-only and probe-only) from the two-color signal on a pulse-to-pulse basis.

The photoelectrons are guided into a time-of-flight spectrometer by a magnetic bottle and detected using a chevron-type 
MCP-detector. Calibration with nitric oxide gas transforms the measured TOF-spectra into electron kinetic energy. Further details on the experimental setup and calibration can be found elsewhere. $^{38}$

The sample solution contains $1 \mathrm{mM}$ of adenine or adenosine and $1 \mathrm{mM}$ of a pH 8 buffer made of tris(hydroxymethyl)aminomethane (TRIS) and hydrochloric acid. Furthermore, we added $30 \mathrm{mM}$ sodium chloride to prevent charging of the liquid jet. ${ }^{39}$ The sample solutions are prepared using demineralized water (residual conductivity $=0.25 \mu \mathrm{S}$ ). DNA bases, nucleosides, and TRIS were purchased from Sigma-Aldrich Co. and sodium chloride was purchased from Merck. All chemicals were used without further purification. At the given pulse intensities the sodium chloride does not contribute significantly to the photoelectron spectra as evident from spectra for a sample solution without base or nucleoside (cf. Fig. 4, 8a and 9a).

\section{Results and discussion}

Fig. 3 shows the time-resolved photoelectron spectra of adenine (a) and adenosine (b) in aqueous solution. These spectra reveal the time-dependent kinetic energy distribution of the photoelectrons and the time-dependent population of the excited state. At positive delays, molecules are excited by $266 \mathrm{~nm}$ light and photoionized by $238 \mathrm{~nm}$ light (at negative delays: vice versa). Around the temporal overlap (delay $\sim 0 \mathrm{ps}$ ), we observe a strong and short-lived photoelectron signal for both molecules. The kinetic energy distribution is very broad and covers a range larger than $2 \mathrm{eV}$. In the case of adenine (Fig. 3a), a significant fraction of the signal persists for several ps in the positive delay direction, i.e. after excitation with $266 \mathrm{~nm}$ light. This longer-lived signal is due to the $7 H$ tautomeric form of adenine present in solution, while the short-lived signal is due to the $9 H$ tautomer common to both molecules. ${ }^{19}$

\subsection{One-color two-photon photoionization of adenosine}

For further analysis of the time-dependent spectra, it is very helpful, firstly to understand the one-color spectra. For simplicity, the examination of the one-color spectra will focus on

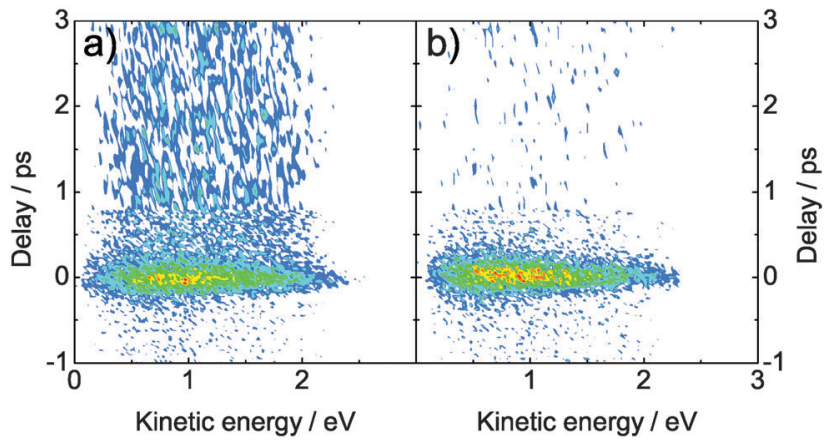

Fig. 3 Time-resolved photoelectron spectra of (a) adenine and (b) adenosine. The molecules were excited with a $266 \mathrm{~nm}$ pulse (4.66 eV photon energy) and probed by $238 \mathrm{~nm}$ ( $5.21 \mathrm{eV}$ photon energy). The one-color multi-photon signals have been subtracted.

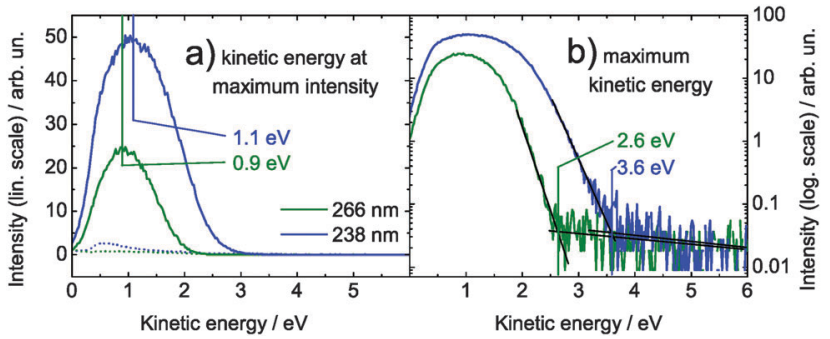

Fig. 4 One-color, two-photon photoelectron spectra of $1 \mathrm{mM}$ adenosine solution for $266 \mathrm{~nm}$ (green) and $238 \mathrm{~nm}$ (blue), corresponding to 4.66 and $5.21 \mathrm{eV}$, respectively. For each spectrum, the photoelectron band maximum (a) and the maximum kinetic energy (b) are identified. The dotted lines in (a) show the one-color photoelectron spectra of the buffer- $\mathrm{NaCl}$ solution for $266 \mathrm{~nm}$ and $248 \mathrm{~nm}$ (pulse energies were almost twice as high as for Ado). Note that the ordinate in (b) is shown on a logarithmic scale.

adenosine because of the presence of only one tautomer. A vertical ionization energy of $7.5 \mathrm{eV}$ for Ade and Ado in aqueous solution is inferred from EUV photoelectron spectroscopy. ${ }^{33}$ For a two-photon ionization process with 4.66 and $5.21 \mathrm{eV}$ pulses, we would therefore expect the photoelectron band maximum at about 1.8 and $2.9 \mathrm{eV}$, respectively. In Fig. 4(a) we show the corresponding one-color two-photon photoelectron spectra of adenosine solution. The photoelectron band maximum is at 0.9 and $1.1 \mathrm{eV}$ electron kinetic energy for twophoton ionization with 4.66 and $5.21 \mathrm{eV}$, respectively. This is significantly lower than the expected values. It is also remarkable that the positions of the band maxima do not differ by the difference in absorbed photon energy, i.e. $2 \times(5.21-4.66) \mathrm{eV}=$ $1.10 \mathrm{eV}$, but only by $0.2 \mathrm{eV}$. In Fig. 4(b), we show the one-color spectra on a logarithmic scale. It is evident that the photoelectron spectrum for the $5.21 \mathrm{eV}$ pulse extends to significantly higher kinetic energies than the spectrum for the $4.66 \mathrm{eV}$ pulse. We define the maximum kinetic energy as the intersection point between the high-energy tail of the spectrum and the noise level, and find $2.6 \mathrm{eV}$ and $3.6 \mathrm{eV}$ for the $4.66 \mathrm{eV}$ and $5.21 \mathrm{eV}$ pulses, respectively. In contrast to the position of the band maxima, the difference between the maximum kinetic energies agrees well with the difference in absorbed photon energy.

We explain these observations by resonance enhanced 2-photon photoionization and initial dynamics of the excited state occurring within the pulse duration of $\sim 80$ fs. The situation is sketched in Fig. 7(a) and (b). Absorption of a first photon prepares the excited state. The Franck-Condon region for the excitation step is indicated by a colored rectangle. For infinitely short pulses or negligible initial dynamics, we expect ionization from the Franck-Condon region. However, if the excited state evolves within the pulse duration, the second photon may ionize the molecule after nuclear reconfiguration has already started. This will result in lower photoelectron kinetic energies and photoelectron bands which are significantly broader than supported by the initial Franck-Condon region. As a result of the initial dynamics, the maximum of the photoelectron band cannot be related to the 1-photon ionization energy. The vertical ionization energy will be somewhat smaller than that derived from the band maximum. The maximum 
kinetic energy observed in the experiment can be assigned to the adiabatic (0-0) transition from the ground state $S_{0}$ to the ionic state $\mathrm{D}_{0}$. We obtained an adiabatic $(0-0)$ transition energy of $(6.82 \pm 0.15) \mathrm{eV}$ with $5.21 \mathrm{eV}$ pulses and $(6.72 \pm 0.15) \mathrm{eV}$ with $4.66 \mathrm{eV}$ pulses. Both values are in satisfactory agreement with the calculated value $6.6 \mathrm{eV} .^{34}$ For the photoelectron spectrum obtained with $5.21 \mathrm{eV}$ pulses (Fig. 7(b)), higher excited states $S_{n}$ are populated by absorption of a first photon. These will decay to $\mathrm{S}_{1}$ within $40 \mathrm{fs}^{26}{ }^{26}$ Along with the higher excess energy, this will significantly broaden the photoelectron band.

\subsection{Time-dependent spectra of excited adenosine}

Fig. 5 shows the pump-probe photoelectron spectrum of adenosine solution around the temporal overlap of the pulses, i.e. averaged over all spectra acquired at delays of $(0 \pm 100)$ fs. At the temporal overlap, we expect ionization after absorption of one photon from each pulse. Hence, the kinetic energy of the photoelectrons should be around $(4.66+5.21-7.5) \mathrm{eV}=$ $2.37 \mathrm{eV}$. As observed for the one-color spectra, the measured photoelectron band maximum $(0.9 \mathrm{eV})$ is much lower than expected. From the maximum kinetic energy of $3.0 \mathrm{eV}$, we extract a value for the adiabatic $(0-0)$ ionization potential of $(6.87 \pm 0.15) \mathrm{eV}$, again in reasonable agreement with the theoretical value of $6.6 \mathrm{eV} .^{34}$

Fig. 6 shows the photoelectron spectrum at different delays for adenosine solution, (a), (c) excited with $4.66 \mathrm{eV}$ and ionized with $5.21 \mathrm{eV}$, and (b), (d) vice versa. Fig. 6(a) and (b) show the raw spectra (each averaged over $\pm 0.1 \mathrm{ps}$ ) with a fit to the function of type $y=A x \mathrm{e}^{\left(-B x^{2}\right)}$. In Fig. 6(c) and (d) the fit maximum is scaled to 1 to illustrate the shift of the spectral weight and narrowing of the photoelectron band with increasing delay. The spectral shape of the photoelectron bands is intricate. Therefore, we describe spectral shifts in terms of the average kinetic energy (AKE)

$$
\mathrm{AKE}=\frac{\int_{0}^{\infty} E_{\mathrm{kin}} I\left(E_{\mathrm{kin}}\right) \mathrm{d} E_{\mathrm{kin}}}{\int_{0}^{\infty} I\left(E_{\mathrm{kin}}\right) \mathrm{d} E_{\mathrm{kin}}}
$$

where $I\left(E_{\text {kin }}\right)$ is the intensity of the signal at the kinetic energy $E_{\text {kin. }}$. Upon excitation with $4.66 \mathrm{eV}$, the AKE shifts only slightly from $1.10 \mathrm{eV}$ at $0 \mathrm{ps}$ to $1.02 \mathrm{eV}$ at $0.2 \mathrm{ps}$ and to $0.95 \mathrm{eV}$ at $0.3 \mathrm{ps}$. In total, we observe an average shift of $150 \mathrm{meV}$ within $300 \mathrm{fs}$. This shift is visualized in Fig. 7(c) for time delays of 0 ps and 0.3 ps.

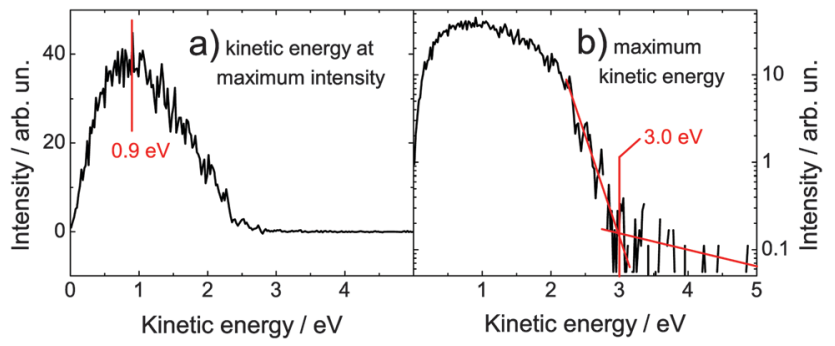

Fig. 5 Time-resolved spectrum of adenosine solution at the temporal overlap $( \pm 0.1 \mathrm{ps})$ of both pulses ( 4.66 and $5.21 \mathrm{eV}$ ). The maximum kinetic energy is $3.0 \mathrm{eV}$. The one-color multi-photon signals have been subtracted. Note that the ordinate in (b) is scaled logarithmically.

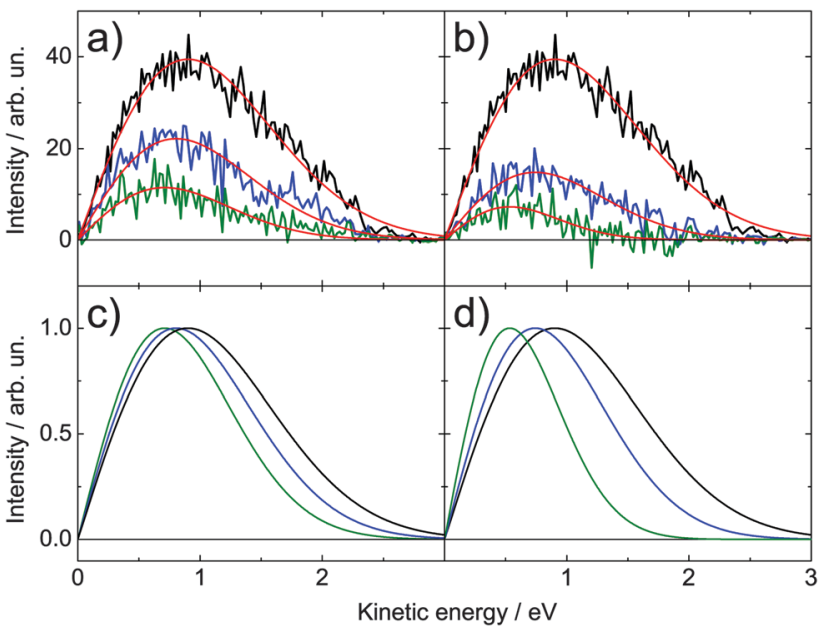

Fig. 6 Time-resolved spectra of adenosine (a) for excitation with $4.66 \mathrm{eV}$ and ionization with $5.21 \mathrm{eV}$ and (b) vice versa. In both cases, the spectra at delay times of 0 ps (black), 0.2 ps (blue) and 0.3 ps (green) are shown. Note that each spectrum is averaged over $\pm 0.1 \mathrm{ps}$. These spectra were fitted with a function of the type $y=A x \mathrm{e}^{-B x^{2}}$. Sub-figures (c) and (d) show these fits scaled to the maximum of 1 .

The excited state is prepared in the same way as for the one-color signal, namely by absorption of a $4.66 \mathrm{eV}$ photon (indicated by the same colored rectangle). Initial dynamics broadens the photoelectron band and subsequent evolution of the excited state leads to a time-dependent shift of the AKE.

After excitation with $5.21 \mathrm{eV}$ pulses, the AKE changes faster: $0.95 \mathrm{eV}$ at $0.2 \mathrm{ps}$ and $0.73 \mathrm{eV}$ at $0.3 \mathrm{ps}$. This shift of $370 \mathrm{meV}$ within 300 fs is illustrated in Fig. 7(d). Absorption of the first photon leads to an excited state higher than $\mathrm{S}_{1}$. Therefore, dynamics are accelerated by the initial $S_{n} \rightarrow S_{1}$ transition accompanied by a generation of vibrational excess energy reducing the average ionization energy.

\subsection{Population dynamics of adenosine and adenine}

For a quantitative analysis of the excited state lifetimes, the photoelectron signal is integrated over all kinetic energies and plotted versus delay time. In Fig. 8, such spectrally integrated signals at different probe wavelengths $(248,243$ and $238 \mathrm{~nm})$ are shown for adenosine. For each temporal direction, these time-dependent signals are fitted by a convolution of a sum of exponential decays and a Gaussian cross correlation function between pump and probe pulses. The cross correlation width was estimated to be 0.1 ps. The time traces are treated as a sum (red) of two components: one towards positive delays (green) and one towards negative delays (blue). The results of these fits are summarized in Table 1.

For positive delays, the molecules are excited by the $266 \mathrm{~nm}$ pulse in each case. If the detected dynamics were limited by the accessibility of the Franck-Condon window of the ionization step, we would find longer lifetimes for larger probe photon energies. However, this is not true for adenosine. For $4.66 \mathrm{eV}$ excitation, the extracted lifetimes $\tau$ are equal within the error bars (see Table 1). The averaged lifetime of $(215 \pm 20) \mathrm{fs}$ is in 

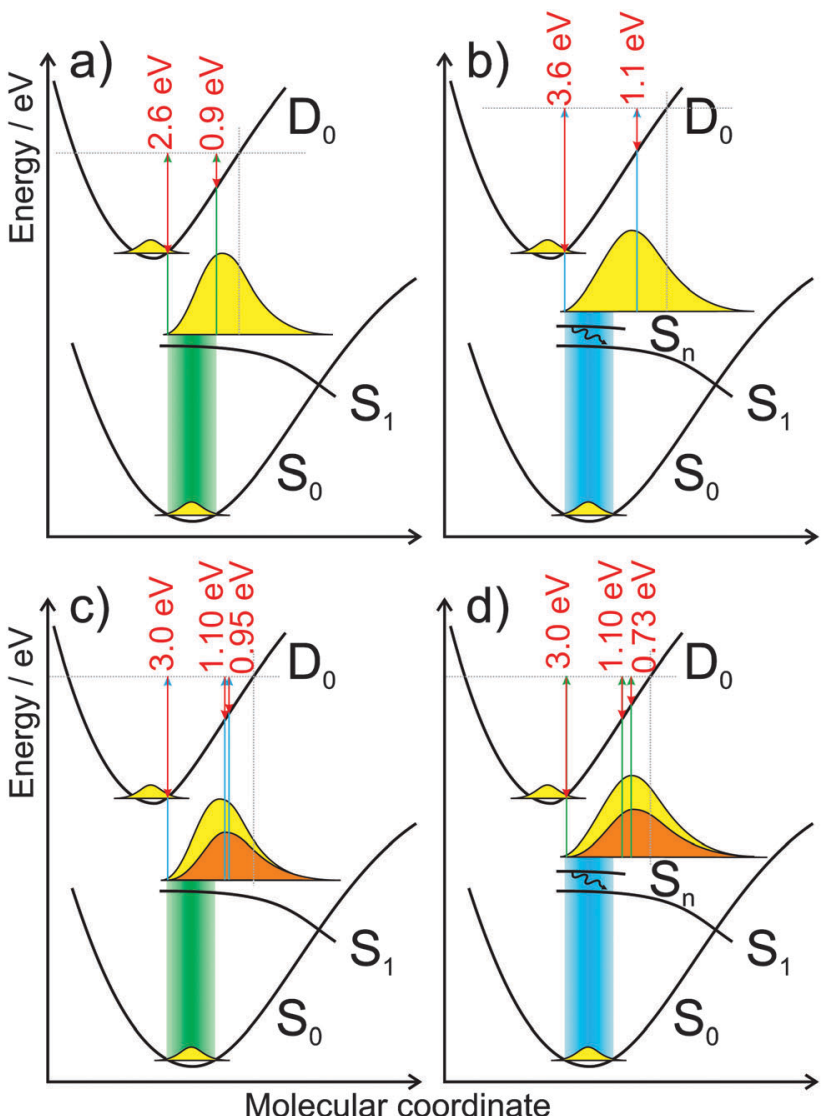

Fig. 7 Potential energy curves from Fig. 2 adapted to the one-color two-photon ionization with (a) $4.66 \mathrm{eV}$ and (b) $5.21 \mathrm{eV}$ pulses and the pump probe processes (c and d). For the pump probe process: (c) excitation with $4.66 \mathrm{eV}$ pulse and ionization with $5.21 \mathrm{eV}$, and (d) vice versa. Excitation is indicated by a broad colored area. The predicted 40 fs decay from $S_{n}$ to $S_{1}$ is indicated by a black arrow ((b) and (d)). The yellow shape symbolizes the excited moiety at the end of the pulse, when it is broadened and has started to relax. For the ionization step, the maximum kinetic energy and the photoelectron band maxima are shown by the red arrows (for the pump-probe process at delay times $0 \mathrm{ps}$ and $0.3 \mathrm{ps}$ ). The yellow shape symbolizes the excited moiety at pump probe delay $0 \mathrm{ps}$, the orange shape at $0.3 \mathrm{ps}$. Grey horizontal lines mark the sum of the photon energies and grey vertical lines indicate the end of the Franck-Condon window for ionization.

the range of earlier results obtained from transient absorption by Pecourt et al. $^{21}$ and fluorescence up-conversion by Onidas et al., ${ }^{22}$
Table 1 Lifetimes of adenosine and adenine

\begin{tabular}{|c|c|c|c|c|}
\hline \multicolumn{2}{|c|}{ Photon energies/eV } & \multirow{2}{*}{$\begin{array}{l}\text { Adenosine } \\
\tau / \text { fs }\end{array}$} & \multicolumn{2}{|c|}{ Adenine } \\
\hline Pump & Probe & & $\tau_{1} / \mathrm{fs}$ & $\tau_{2} / \mathrm{ps}$ \\
\hline 4.66 & 5.00 & $250 \pm 40$ & $81 \pm 7$ & $2.8 \pm 0.9$ \\
\hline 4.66 & 5.10 & $210 \pm 20$ & $76 \pm 5$ & $2.4 \pm 0.8$ \\
\hline 4.66 & 5.21 & $210 \pm 20$ & $64 \pm 2$ & $8.5 \pm 1.7$ \\
\hline 5.00 & 4.66 & $143 \pm 6$ & & \\
\hline 5.10 & 4.66 & $125 \pm 7$ & & \\
\hline 5.21 & 4.66 & $97 \pm 5$ & & \\
\hline
\end{tabular}

who found $(290 \pm 40)$ fs and $(170 \pm 10)$ fs, respectively. Pancur et al. found a somewhat larger value of $(310 \pm 50)$ fs for the excited state lifetime from their fluorescence up-conversion measurements. ${ }^{20}$

At negative delays, dynamics are initiated by pump pulses with photon energies between 5.00 and $5.21 \mathrm{eV}$ and probed by $4.66 \mathrm{eV}$ pulses. The excess energy introduced into the excited state rises when going from $5.00 \mathrm{eV}$ in Fig. 8 (a) to $5.21 \mathrm{eV}$ in Fig. $8(\mathrm{c})$. With increasing excess energy in the $\mathrm{S}_{1}$ state the conical intersection to $\mathrm{S}_{0}$ can be reached in a shorter time; furthermore, also higher lying conical intersections may play an increasing role. Thus, a smaller time constant is expected. This effect is revealed by the extracted lifetimes $\tau$ : They decrease significantly from $\sim 140 \mathrm{fs}$ for $5.00 \mathrm{eV}$ excitation to $\sim 100 \mathrm{fs}$ for $5.21 \mathrm{eV}$ excitation. Similar behavior was found by Pancur et al. ${ }^{20}$ who measured fluorescence up-conversion of adenosine using tunable UV pump pulses. They found slightly shorter fluorescence lifetimes for excitation with $<260 \mathrm{~nm}$ pulses compared to excitation with $>260 \mathrm{~nm}$ pulses.

In Fig. 9, the time-dependent signal for adenine is shown for the same wavelength combinations. Compared to adenosine, a second time constant appears after excitation with $4.66 \mathrm{eV}$ which is due to the $7 H$ tautomer. ${ }^{19}$ This second lifetime seems to depend on the probe wavelength. With increasing probe photon energy the measured lifetime increases. As explained before, this behavior can be attributed to a longer residence within the Franck-Condon window for higher probe photon energies. It is interesting to note that our lifetime $\tau_{2}$ of $(8.5 \pm 1.7) \mathrm{ps}$ obtained for the highest probe photon energy $(5.21 \mathrm{eV})$ is in excellent agreement with the value of $(8.4 \pm 0.8)$ ps measured in time-resolved fluorescence up-conversion experiments. ${ }^{20}$
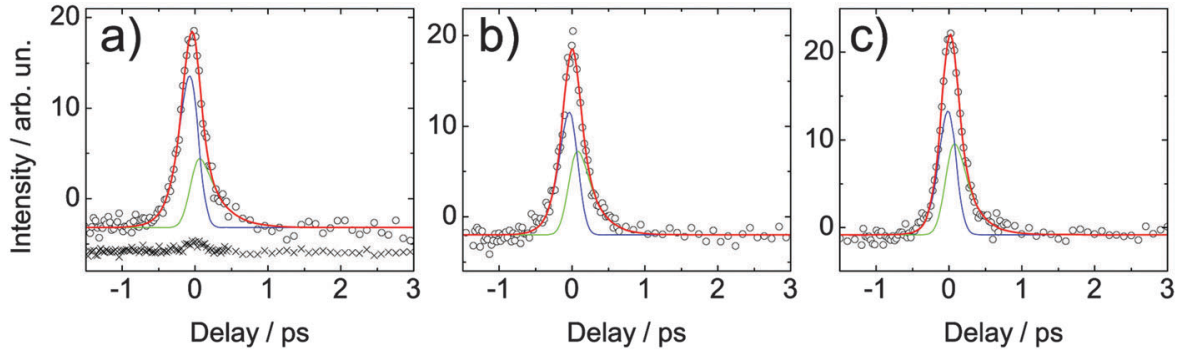

Fig. 8 Spectrally integrated signal of adenosine in aqueous solution measured at different wavelengths. The solution was excited by a $266 \mathrm{~nm}$ ( $4.66 \mathrm{eV}$ ) pulse and probed by (a) $248 \mathrm{~nm}(5.00 \mathrm{eV})$, (b) $243 \mathrm{~nm}(5.10 \mathrm{eV})$ and (c) $238 \mathrm{~nm}(5.21 \mathrm{eV})$. Open circles are data points, the blue and green lines fit the population dynamics in the negative and the positive delay direction, respectively. The cumulative fit is shown in red. The lifetimes extracted from these fits are shown in Table 1. For comparison, also the signal of the buffer- $\mathrm{NaCl}$ solution is shown (crosses). It was measured at the same wavelength but at twice the pulse energy. For clarity, this signal is displaced vertically. 

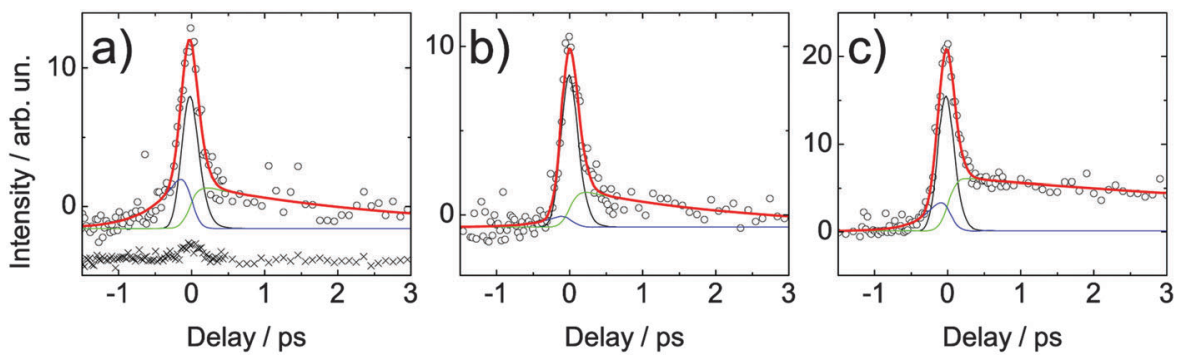

Fig. 9 Spectrally integrated signal of adenine in aqueous solution. The solution was excited with a $266 \mathrm{~nm}(4.66 \mathrm{eV}$ ) pulse and probed by (a) $248 \mathrm{~nm}$ ( $5.00 \mathrm{eV}$ ), (b) $243 \mathrm{~nm}(5.10 \mathrm{eV})$ and (c) $238 \mathrm{~nm}(5.21 \mathrm{eV})$. The crosses in (a) depict the signal of the buffer-NaCl solution which is vertically offset for clarity. It was measured at the same wavelength but almost twice as high pulse energy. For clarity, the buffer signal is vertically displaced.

Thus, for the $5.21 \mathrm{eV}$ probe photons, the Franck-Condon window for ionization is reached during the whole dynamics in the excited electronic state.

At negative delays (for excitation with 5.0-5.2 eV pulses), we also expect to find this second lifetime for the $7 \mathrm{H}$-adenine. The data, however, do not allow for a meaningful bi-exponential fit. Here, only a mono-exponential fit was applied yielding an average lifetime of $(420 \pm 160),(340 \pm 650)$ and $(270 \pm 130) \mathrm{fs}$ for excitation with 5.0, 5.1, and $5.2 \mathrm{eV}$ pulses, respectively. The physical meaning of this single lifetime is very limited, since it represents two lifetimes and, additionally, it is afflicted with huge errors. Nevertheless, this average lifetime is much shorter than expected for the $7 \mathrm{H}$-tautomer. We suppose that for a relatively low probe photon energy of $4.66 \mathrm{eV}$, the FranckCondon window for photoionization is left already in the subpicosecond region.

\section{Conclusions}

Using adenine and adenosine as examples, we have shown that time-resolved photoelectron spectroscopy using probe pulses in the UV range can be used to study the excited state dynamics of molecules in solution. The evolution of the excited state on the potential energy surface could be followed in time, even though significant nuclear rearrangement occurs already within our pulse duration of about $80 \mathrm{fs}$. Here, a significantly higher timeresolution would be desirable. However, different schemes for the UV generation would be required to achieve significantly shorter pulses in this spectral regime (i.e. non-collinear optical parametric amplification ${ }^{40}$ or four-wave mixing processes ${ }^{41}$ ).

The excited state binding energy of adenosine, i.e. the energetic difference between the excited state and the ionic state strongly changes in time. Within $300 \mathrm{fs}$, the binding energy has increased by $150 \mathrm{meV}$ and by $370 \mathrm{meV}$ upon excitation with $266 \mathrm{~nm}$ and $238 \mathrm{~nm}$ pulses, respectively. This is in line with accelerated dynamics initiated by the higher excess energy introduced by the $238 \mathrm{~nm}$ pulses.

The time-dependent energy-integrated photoelectron signal describes the population of the excited state. Depending on the excitation conditions we observe lifetimes in the range of (100-215) fs for adenosine which is in agreement with transient absorption and fluorescence up-conversion data. ${ }^{18-22}$ However, in contrast to previous experiments, we do not probe the excited state in a small range of excited state geometries only. Our probe wavelength dependent measurement does not show any variation in the retrieved excited state lifetime for adenosine within experimental precision and therefore strongly indicates that we indeed observe the relevant features of the excited state evolution. The observed lifetimes are not limited by the accessibility of the Franck-Condon window in the ionization step.

We hope that our experimental work will trigger more theoretical investigations and will help to get deeper insight into the relevant relaxation pathways of the molecules in solution.

\section{Acknowledgements}

The authors thank F. Noack for his support by providing the laser system in the femtosecond application laboratory of the Max-Born-Institut Berlin. This work was financially supported by Deutsche Forschungsgemeinschaft, Project LU 1638/1-1.

\section{References}

1 C. E. Crespo-Hernández, B. Cohen, P. M. Hare and B. Kohler, Chem. Rev., 2004, 104, 1977.

2 L. Serrano-Andrés and M. Merchán, J. Photochem. Photobiol., C, 2009, 10, 21.

3 L. Serrano-Andrés, M. Merchán and A. C. Borin, Proc. Natl. Acad. Sci. U. S. A., 2006, 103, 8691.

4 L. Blancafort, B. Cohen, P. M. Hare, B. Kohler and M. A. Robb, J. Phys. Chem. A, 2005, 109, 4431.

5 M. Barbatti and H. Lischka, J. Am. Chem. Soc., 2008, 6831, 130. 6 A. L. Sobolewski, W. Domcke, C. Dedonder-Lardeux and C. Jouvet, Phys. Chem. Chem. Phys., 2002, 4, 1093.

7 S. Ullrich, T. Schultz, M. Z. Zgierski and A. Stolow, J. Am. Chem. Soc., 2004, 126, 2262.

8 M. Zierhut, W. Roth and I. Fischer, Phys. Chem. Chem. Phys., 2004, 6, 5178.

9 A. Lübcke, F. Buchner, N. Heine, I. V. Hertel and T. Schultz, Phys. Chem. Chem. Phys., 2010, 12, 14629.

10 Y. Tang, H. Shen, K. Sekiguchi, N. Kurahashi, T. Mizuno, Y.-I. Suzuki and T. Suzuki, Phys. Chem. Chem. Phys., 2010, 12, 3653.

11 A. Holmén and A. Broo, Int. J. Quantum Chem., 1995, 22, 113. 12 J. Gu and J. Leszczynski, J. Phys. Chem. A, 1999, 103, 2744. 
13 A. Laxer, D. T. Major, H. E. Gottlieb and B. Fischer, J. Org. Chem., 2001, 66, 5463.

14 M. Dreyfus, G. Dodin, O. Bensaude and J. E. Dubois, J. Am. Chem. Soc., 1975, 97, 2369.

15 M. T. Chenon, R. J. Pugmire, D. M. Grant, R. P. Panzica and L. Townsend, J. Am. Chem. Soc., 1975, 97, 4636.

16 N. C. Gonnella, H. Nakanishi, J. B. Holtwick, D. S. Horowitz, K. Kanamori, N. J. Leonard and J. D. Roberts, J. Am. Chem. Soc., 1983, 105, 2050.

17 A. Holmén, J. Phys. Chem. A, 1997, 101, 4361.

18 T. Gustavsson, A. Sharonov, D. Onidas and D. Markovitsi, Chem. Phys. Lett., 2002, 356, 49.

19 B. Cohen, P. M. Hare and B. Kohler, J. Am. Chem. Soc., 2003, 125, 13594.

20 T. Pancur, N. K. Schwalb, F. Renth and F. Temps, Chem. Phys., 2005, 313, 199.

21 J.-M. L. Pecourt, J. Peon and B. Kohler, J. Am. Chem. Soc., 2001, 123, 10370.

22 D. Onidas, D. Markovitsi, S. Marguet, A. Sharonov and T. Gustavsson, J. Phys. Chem. B, 2002, 106, 11367.

23 C. M. Marian, J. Chem. Phys., 2005, 122, 104314.

24 S. Perun, A. L. Sobolewski and W. Domcke, J. Am. Chem. Soc., 2005, 127, 6257.

25 M. Barbatti, Z. Lan, R. Crespo-Otero, J. J. Szymczak, H. Lischka and W. Thiel, J. Chem. Phys., 2012, 137, 22 A503.

26 Z. Lan, Y. Lu, E. Fabiano and W. Thiel, ChemPhysChem, 2011, 12, 1989.
27 J. C. A. Boeyens, J. Cryst. Mol. Struct., 1978, 8, 317.

28 B. Mennucci, A. Toniolo and J. Tomasi, J. Phys. Chem. A, 2001, 105, 4749.

29 S. Yamazaki and S. Kato, J. Am. Chem. Soc., 2007, 129, 2901.

30 V. Ludwig, Z. M. da Costa, M. S. do Amaral, A. C. Borin, S. Canuto and L. Serrano-Andrés, Chem. Phys. Lett., 2010, 492, 164.

31 R. Mitrić, U. Werner, M. Wohlgemuth, G. Seifert and V. Bonačić-Koutecký, J. Phys. Chem. A, 2009, 113, 12700.

32 C. M. Marian, M. Kleinschmidt and J. Tatchen, Chem. Phys., 2008, 347, 346.

33 R. Seidel, PhD thesis, Technische Universitaet Berlin, 2011.

34 E. Pluhařová, P. Jungwirth, S. E. Bradforth and P. Slaviček, J. Phys. Chem. B, 2011, 115, 1294, ESI†.

35 L. Blancafort, J. Am. Chem. Soc., 2006, 128, 210.

36 M. Faubel, B. Steiner and J. P. Toennies, J. Chem. Phys., 1997, 106, 9013.

37 D. Voet, W. B. Gratzer, R. A. Cox and P. Doty, Biopolymers, 1963, 1, 193.

38 F. Buchner, A. Lübcke, N. Heine and T. Schultz, Rev. Sci. Instrum., 2010, 81, 113107.

39 N. Preissler, F. Buchner, T. Schultz and A. Lübcke, J. Phys. Chem. B, 2013, 117, 2422.

40 L. Gundlach, R. Ernstorfer, E. Riedle, R. Eichberger and F. Willig, Appl. Phys. B: Lasers Opt., 2005, 80, 727.

41 T. Fuji, T. Horio and T. Suzuki, Opt. Lett., 2007, 32, 2481. 